\title{
Degenerate Crossing Numbers
}

\author{
János Pach • Géza Tóth
}

Received: 24 July 2006 / Revised: 17 April 2007 / Accepted: 17 April 2007 /

Published online: 14 February 2009

(C) Springer Science+Business Media, LLC 2009

\begin{abstract}
Let $G$ be a graph with $n$ vertices and $e \geq 4 n$ edges, drawn in the plane in such a way that if two or more edges (arcs) share an interior point $p$, then they properly cross one another at $p$. It is shown that the number of crossing points, counted without multiplicity, is at least constant times $e$ and that the order of magnitude of this bound cannot be improved. If, in addition, two edges are allowed to cross only at most once, then the number of crossing points must exceed constant times $(e / n)^{4}$.
\end{abstract}

Keywords Crossing number - Crossing lemma - Bisection width · Euler characteristics $\cdot$ Incidences $\cdot$ Multiple crossings

\section{Introduction}

Let $S$ be a compact surface with no boundary. Given a graph $G$ with no loops or multiple edges, the crossing number of $G$ on $S$, denoted by $\mathrm{CR}_{S}(G)$, is the minimum number of edge crossings over all proper drawings of $G$ on $S$. If $S$ is the sphere (or plane), then we simply write $\mathrm{CR}(G)$. A drawing is proper if the vertices and edges of $G$ are represented by points and simple Jordan-arcs in $S$ such that no arc representing an edge passes through a point representing a vertex other than its endpoints. Here we count a $k$-fold crossing $\left(\begin{array}{l}k \\ 2\end{array}\right)$ times (or, equivalently, no three edges can pass through

The research of J. Pach was supported by NSF grant CCF-05-14079 and by grants from NSA, PSC-CUNY, BSF, and OTKA-K-60427.

The research of G. Tóth was supported by OTKA-K-60427.

J. Pach $(\bowtie) \cdot$ G. Tóth

Rényi Institute, Hungarian Academy of Sciences, Budapest, Hungary

e-mail: pach@cims.nyu.edu

J. Pach

EPFL, Lausanne, Switzerland 
Fig. $1 \mathrm{CR}(G)=2, \mathrm{CR}^{*}(G)=1$
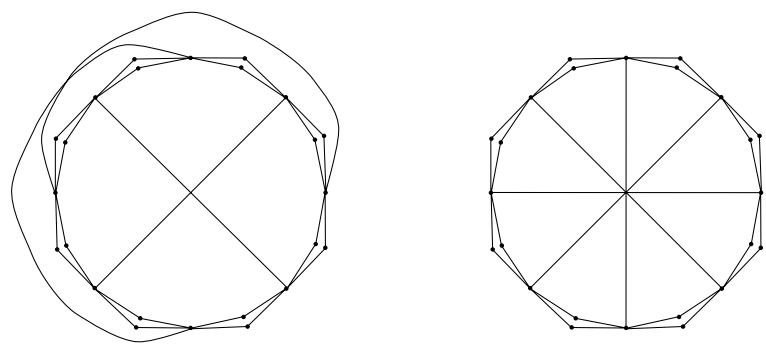

the same point). We also assume that between the arcs no tangencies are allowed. See [6] for a survey.

G. Rote, M. Sharir, and others asked what happens if multiple crossings are counted only once (equivalently, if several edges are allowed to pass through the same point)? To what extent does this modification effect the notion of crossing number?

Let $\mathrm{CR}^{*}(G)$ denote the degenerate crossing number of $G$, that is, the minimum number of crossing points over all drawings of $G$, where $k$-fold crossings are also allowed. Of course, we have

$$
\mathrm{CR}^{*}(G) \leq \mathrm{CR}(G)
$$

and the two crossing numbers are not necessarily equal. For example, in the plane Kleitman [2] proved that the crossing number of the complete bipartite graph $K_{5,5}$ with five vertices in its classes is 16 . On the other hand, the degenerate crossing number of $K_{5,5}$ in the plane is at most 15. Another example is depicted in Fig. 1.

Let $n=n(G)$ and $e=e(G)$ denote the number of vertices and the number of edges of a graph $G$. Ajtai, Chvátal, Newborn, and Szemerédi [1] and, independently, Leighton [3] proved that

$$
\mathrm{CR}(G) \geq \frac{1}{64} \frac{e^{3}(G)}{n^{2}(G)}
$$

for every graph $G$ with $e(G) \geq 4 n(G)$. This statement, which has many interesting applications in combinatorial geometry, easily generalizes to crossing numbers of graphs drawn on any fixed surface $S$ (see [8]).

In the present note we investigate whether the above inequality remains true for the degenerate crossing number of $G$. First, we show that the answer is "no" if we permit drawings in which two edges may cross an arbitrary number of times.

Theorem 1.1 Any graph with $n$ vertices and e edges has a proper drawing in the plane with fewer than e crossings, where each crossing point that belongs to the interior of several edges is counted only once. The order of magnitude of this bound cannot be improved if $e \geq 4 n$.

Therefore, in Sect. 3 we restrict our attention to so-called simple drawings, i.e., to proper drawings in which two edges are allowed to cross at most once. From now on, with a slight abuse of notation, $\mathrm{CR}^{*}(G)$ will stand for the minimum number of crossings over all simple drawings.

We want to decide whether $\mathrm{CR}^{*}(G)=\Omega\left(e^{3} / n^{2}\right)$ holds for all graphs with $e \geq 4 n$. Theorem 1.1 implies that this is the case for "sparse" graphs, that is, for graphs with 
$e=O(n)$. The next theorem shows that the statement is also true for very "dense" graphs having a quadratic number of edges. More precisely, we have the following:

Theorem 1.2 There exists a constant $c^{*}>0$ such that the degenerate crossing number of $G$ satisfies

$$
\mathrm{CR}^{*}(G) \geq c^{*} \frac{e^{4}(G)}{n^{4}(G)}
$$

for any graph $G$ with $e(G) \geq 4 n(G)$.

If it causes no confusion, in notation and terminology we make no distinction between the graph $G$ and its drawing, and between a vertex (edge) and the point (arc) representing it.

\section{Proper Drawings with Few Crossings}

In this section we prove Theorem 1.1.

Let $\pi=(\pi(1), \pi(2), \ldots, \pi(e))$ be a permutation of the first $e$ positive integers, and let $1 \leq i<j \leq e$. Reversing the order of the elements between $\pi(i)$ and $\pi(j)$, we obtain another permutation

$$
\begin{aligned}
\pi^{\prime}= & (\pi(1), \pi(2), \ldots, \pi(i-1), \pi(j), \pi(j-1), \ldots, \pi(i), \pi(j+1), \\
& \pi(j+2), \ldots, \pi(e)) .
\end{aligned}
$$

Such an operation is called a swap.

Lemma 2.1 Any permutation of e numbers can be obtained from any other permutation by performing at most e -1 swaps.

Proof The proof is by induction on $e$. For $e=1$, the statement is trivial. Suppose that the lemma has been verified for permutations of fewer than $e$ numbers. Let $\sigma=(\sigma(1), \sigma(2), \ldots, \sigma(e))$ and $\pi=(\pi(1), \pi(2), \ldots, \pi(e))$ be two permutations of size $e$. For some $j$, we have $\pi(j)=\sigma(e)$. To obtain $\sigma$ from $\pi$, we first swap the interval $(\pi(j), \ldots, \pi(e))$ of $\pi$. The last element of the resulting permutation $(\pi(1), \pi(2)$, $\ldots, \pi(j-1), \pi(e), \pi(e-1), \ldots, \pi(j))$ is now the same as the last element of the target permutation $\sigma$. Proceeding by induction, we can attain using at most $e-2$ further swaps that all elements coincide.

Proof of Theorem 1.1 Let $G$ be a graph with $e$ edges and $n$ vertices, $v_{1}, v_{2}, \ldots, v_{n}$. Arbitrarily orient every edge of $G$. For $1 \leq i \leq n$, place $v_{i}$ at the point $(0, i)$ on the $y$-axis. Each edge will be drawn as a continuous arc running close to a huge circle centered at a faraway point of the positive $y$-axis, so that its initial and final portions are almost horizontal segments, oriented from left to right, that belong to the halfplanes $x \geq 0$ and $x \leq 0$, respectively. (See Fig. 2.) More precisely, for each edge $\overrightarrow{v_{i} v_{j}}$, draw a short almost horizontal initial segment from $v_{i}$ pointing to the right and a short almost horizontal final segment pointing to $v_{j}$ from the left. Suppose that 
Fig. $2 \mathrm{CR}^{*}(G) \leq e-1$

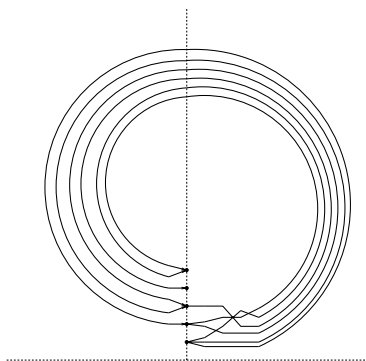

all these segments have different slopes. From bottom to top, enumerate the initial segments by $1,2, \ldots, e$ and assign the same numbers to the final segments of the corresponding edges lying in the negative half-plane $x \leq 0$. The indices of these final segments (from bottom to top) form a permutation $\sigma=(\sigma(1), \sigma(2), \ldots, \sigma(e))$. We have to connect the right endpoint of each initial segment to the left endpoint of the final segment denoted by the same number. These connecting arcs will run parallel to one another, roughly along huge concentric circles, except that at certain points several arcs will cross.

By Lemma 2.1, $\sigma$ can be obtained from $1,2, \ldots, e$ by a sequence of at most $e-1$ swaps. We can "realize" each swap as a crossing of the corresponding arcs at a single point. The participating arcs leave the crossing in reverse order. Thus, introducing at most $e-1$ crossings, we can achieve that the order of the connecting arcs is identical to the order in which their final segments must reach the $y$-axis (from the left).

It follows from Lemma 2.2 (see below) that any proper drawing of $G$ has at least $\frac{e}{3}-n+2$ crossings.

We prove the tightness of Theorem 1.1 in a slightly more general setting. Let $S$ be a compact surface $S$ with no boundary whose Euler characteristic is $\chi$. That is, we have

$$
\chi(S)= \begin{cases}2-2 g & \text { if } S \text { is orientable of genus } g, \\ 2-g & \text { if } S \text { is nonorientable of genus } g .\end{cases}
$$

Given a connected graph $G$ with no loops or multiple edges, let $\underline{\mathrm{CR}}_{S}(G)$ stand for the minimum number of crossing points over all proper drawings of $G$ on $S$. Taking the minimum over all simple drawings (that is, allowing two edges to cross only at most once), we obtain the degenerate crossing number of $G$ on $S$, denoted by $\operatorname{CR}_{S}^{*}(G)$. Clearly, we have $\underline{\mathrm{CR}}_{S}(G) \leq \mathrm{CR}_{S}^{*}(G)$ for any $G$.

Lemma 2.2 Let $G$ be a graph with $n(G)$ vertices and e $(G)$ edges, and let $S$ be a surface with Euler characteristic $\chi$. Then we have

$$
\mathrm{CR}_{S}^{*}(G) \geq \underline{\mathrm{CR}}_{S}(G) \geq \frac{e(G)}{3}-n(G)+\chi .
$$

Proof Fix an optimal proper drawing of $G$ on $S$, i.e., a drawing for which the number of crossings is $\mathrm{CR}_{S}(G)$. Let $p$ be a crossing determined by $k$ edges $e_{1}, e_{2}, \ldots, e_{k}$. Remove from $S$ a small rectangular piece $A B C D$ such that each $e_{i}$ intersects its boundary in two points $A_{i} \in A B$ and $C_{i} \in C D$ and the counterclockwise order of 
these points is $A_{1}, A_{2}, \ldots, A_{k}, C_{1}, C_{2}, \ldots, C_{k}$. Assume that no further edges of $G$ meet the rectangle $A B C D$. Modify $S$ by adding a crosscap at $A B C D$, i.e., by identifying $A_{i}$ and $C_{i}$ for every $i$ (and identifying all other "diametrically opposite" pairs of points of the boundary of $A B C D$ ). In this way, we reduce the number of crossings by one and we obtain a drawing of $G$ on a surface whose Euler characteristic is $\chi(S)-1$. Repeating the same procedure at each crossing, finally we obtain a crossing-free drawing of $G$ on a (nonorientable) surface $S^{\prime}$ with Euler characteristic $\chi(S)-\underline{\mathrm{CR}}_{S}(G)$. The number of faces or cells in this embedding is denoted by $f(G)$.

According to Poincaré's formula, a generalization of Euler's polyhedral formula, we have

$$
n(G)-e(G)+f(G) \geq \chi\left(S^{\prime}\right)=\chi(S)-\underline{\mathrm{CR}}_{S}(G) .
$$

This inequality becomes an equation if the embedding is cellular, that is, if the boundary of each face is connected. For details, see [5]. Taking into account that $3 f(G) \leq 2 e(G)$, we obtain

$$
\underline{\mathrm{CR}}_{S}(G) \geq \frac{e(G)}{3}-n(G)+\chi(S),
$$

as required.

The nonorientable genus $\gamma(G)$ of a graph $G$ is the minimum genus of a nonorientable surface in which $G$ can be embedded with no crossings. Mohar [4] showed that the nonorientable genus is equal to the planar degenerate crossing number, that is, for any simple graph $G$, we have $\gamma(G)=\underline{\mathrm{CR}}_{S}(G)$, where $S$ is the sphere (or the plane).

\section{Simple Drawings: Proof of Theorem 1.2}

Let $\mathrm{CR}^{*}(G)$ stand for the minimum number of crossing points over all simple drawings of $G$ in the plane.

Lemma 3.1 Let $G$ be a graph with $n$ vertices and e edges, and suppose that the crossing number of $G$ satisfies $\mathrm{CR}(G)>10^{3} e(G) n(G)$. Then for the degenerate crossing number of $G$, we have

$$
\mathrm{CR}^{*}(G) \geq \frac{\mathrm{CR}^{3}(G)}{(40 e)^{4}}
$$

Proof Consider a simple drawing of $G$ with $\mathrm{CR}^{*}(G)$ crossing points. Let $M:=$ $40^{2} e^{2} / \mathrm{CR}(G)$.

For any crossing (point) $p$, let $m(p)$ denote the multiplicity of $p$, that is, the number of edges passing through $p$. Let $S$ denote the set of crossings of multiplicity at most $M$. For any integer $i \geq 0$, let $S_{i}$ be the set of crossing points $p$ with $2^{i} M<m(p) \leq 2^{i+1} M$. Since $m(p)$ cannot exceed $n / 2$, we have $S_{i}=\emptyset$ whenever $2^{i} M>n / 2$. It follows from the generalization of the Szemerédi-Trotter theorem 
$[9,10]$ for bounding the number of incidences between a set of points and a set of pseudo-segments that the number of crossings of multiplicity at least $k$ is at most $100\left(\frac{e^{2}}{k^{3}}+\frac{e}{k}\right)$. That is,

$$
\left|S_{i}\right| \leq 100\left(\frac{e^{2}}{2^{3 i} M^{3}}+\frac{e}{2^{i} M}\right)
$$

for every $i$. The number of crossing pairs of edges is at least $\mathrm{CR}(G)$, and each point of multiplicity $k$ contributes $\left(\begin{array}{l}k \\ 2\end{array}\right)<k^{2} / 2$ to this number. Therefore, the total contribution of the points in $S_{i}$ is at most

$$
100\left(\frac{e^{2}}{2^{3 i} M^{3}}+\frac{e}{2^{i} M}\right) 2^{2 i+1} M^{2}=100\left(\frac{e^{2}}{M} 2^{1-i}+e M 2^{i+1}\right) .
$$

Adding up, we obtain that the contribution of all crossings of multiplicity larger than $M$ to the number of crossing pairs of edges is at most

$$
\sum_{\substack{i \geq 0 \\ M 2^{i} \leq n / 2}} 100\left(\frac{e^{2}}{M} 2^{1-i}+e M 2^{i+1}\right)<100\left(\frac{4 e^{2}}{M}+2 e n\right)<\frac{\mathrm{CR}(G)}{2} .
$$

Therefore, at least half of the edge crossings occur at points of multiplicity at most $M$, that is, at a point belonging to $S$. Each of these points contributes to the crossing number at most $\left(\begin{array}{c}M \\ 2\end{array}\right)<\frac{M^{2}}{2}$. Thus, we have $|S| \frac{M^{2}}{2}>\frac{\mathrm{CR}(G)}{2}$, which yields that $|S|>\frac{\mathrm{CR}^{3}(G)}{(40 e)^{4}}$.

In view of the fact that $\mathrm{CR}(G)$ is at least constant times $e^{3} / n^{2}$, Lemma 3.1 already implies that Theorem 1.2 is true for dense graphs: if $G$ has a quadratic number of edges (in $n$ ), then the order of magnitude of the degenerate crossing number $\mathrm{CR}^{*}(G)$ is $n^{4}$.

The bisection width, $b(G)$, of a graph $G$ is defined as the minimum number of edges whose removal splits the graph into two roughly equal subgraphs. More precisely, $b(G)$ is the minimum number of edges running between $V_{1}$ and $V_{2}$, over all partitions of the vertex set of $G$ into two parts $V_{1} \cup V_{2}$ such that $\left|V_{1}\right|,\left|V_{2}\right| \geq n(G) / 3$. We need the following result.

Lemma 3.2 [7] Let $G$ be a graph of $n$ vertices and e edges. Then we have

$$
b(G) \leq 10 \sqrt{\mathrm{CR}(G)}+4 \sqrt{e n} .
$$

For the proof of Theorem 1.2, we pick a nested sequence of subgraphs $G=G_{0} \supset$ $G_{1} \supset G_{2} \supset \cdots$, according to the following procedure.

STEP 0. Set $G_{0}:=G, n_{0}:=n(G)=n, e_{0}:=e(G)=e$, and $\mathrm{CR}_{0}=: \operatorname{CR}(G)$.

Suppose that we have already executed STEP $i$. Denote the resulting graph by $G_{i}$, let $n_{i}=n\left(G_{i}\right), e_{i}=e\left(G_{i}\right), \mathrm{CR}_{i}=\mathrm{CR}\left(G_{i}\right)$, and assume that $(1 / 3)^{i} n \leq n_{i} \leq(2 / 3)^{i} n$. 
STEP $i+1$. If

$$
\mathrm{CR}_{i} \geq\left(\frac{e_{i} e}{n}\right)^{4 / 3}+10^{3} e_{i} n_{i}
$$

then STOP.

Else, delete $b\left(G_{i}\right)$ edges from $G_{i}$ such that $G_{i}$ falls into two parts, both having at most (2/3) $n_{i}$ vertices. Let $G_{i}^{\prime}$ be the resulting (disconnected) graph. Let $G_{i+1}$ be the part in which the average degree of the vertices is at least as high as in the other.

Suppose that the algorithm terminates in STEP $I+1$.

Lemma 3.3 Suppose that $e(G)>n^{4 / 3}(G)$. For any $0 \leq i \leq I$ such that $e_{i} \geq$ $10^{12}(e / n)^{2}$, we have $\frac{e_{i}}{n_{i}}>\frac{e}{2 n}$.

Proof We prove the statement by induction on $i$. Obviously, it is true for $i=0$. Let $1 \leq i \leq I$, and suppose that the lemma has been proved for all $j<i$.

Since the procedure did not stop at an earlier stage, we have

$$
\mathrm{CR}_{j}<\left(\frac{e_{j} e}{n}\right)^{4 / 3}+10^{3} e_{j} n_{j}
$$

for every $j<i$. In view of Lemma 3.2, we obtain

$$
\begin{aligned}
e\left(G_{j}^{\prime}\right) & =e_{j}-b\left(G_{j}\right) \geq e_{j}-10 \sqrt{\mathrm{CR}_{j}}-4 \sqrt{e_{j} n_{j}} \\
& \geq e_{j}\left(1-\frac{10(e / n)^{2 / 3}}{e_{j}^{1 / 3}}-10^{3 / 2} \sqrt{\frac{n_{j}}{e_{j}}}-4 \sqrt{\frac{n_{j}}{e_{j}}}\right) \\
& \geq e_{j}\left(1-\frac{10(e / n)^{2 / 3}}{e_{j}^{1 / 3}}-40 \sqrt{\frac{n_{j}}{e_{j}}}\right) .
\end{aligned}
$$

Using the fact that the average degree in $G_{j+1}$ is at least as much as in $G_{j}^{\prime}$ and that $i \leq 2 \log _{2} n$, we have

$$
\begin{aligned}
\frac{e_{i}}{n_{i}} & \geq \frac{e}{n} \prod_{0 \leq j<i}\left(1-\frac{10(e / n)^{2 / 3}}{e_{j}^{1 / 3}}-40 \sqrt{\frac{n_{j}}{e_{j}}}\right) \\
& \geq \frac{e}{n}\left(1-\sum_{0 \leq j<i} \frac{10(e / n)^{2 / 3}}{e_{j}^{1 / 3}}-\sum_{0 \leq j<i} 40 \sqrt{\frac{n_{j}}{e_{j}}}\right) \\
& \geq \frac{e}{n}\left(1-20(e / n)^{1 / 3} \sum_{0 \leq j<i} \frac{1}{n_{j}^{1 / 3}}-80 \log n \sqrt{\frac{2 n}{e}}\right) \\
& \geq \frac{e}{n}\left(1-200(e / n)^{1 / 3} \cdot \frac{1}{n_{i}^{1 / 3}}-80 \log n \sqrt{\frac{2 n}{e}}\right)>\frac{e}{2 n},
\end{aligned}
$$

provided that $n=n(G)$ is large enough. This concludes the proof of Lemma 3.3. 
Proof of Theorem 1.2 If $e \leq n^{4 / 3}$, then the result is an immediate consequence of Lemma 3.1.

Assume that $e>n^{4 / 3}$ and that the procedure stopped at step $I+1$. We distinguish three cases.

Case 1: Suppose that $e=e_{0}<4 \cdot 10^{12}(e / n)^{2}$. Then $e>n^{2} /\left(4 \cdot 10^{12}\right)$. By the result of Ajtai, Chvátal, Newborn, and Szemerédi [1] and Leighton [3], quoted in Sect. 1 (see above Theorem 1.1), we have

$$
\mathrm{CR}(G) \geq \frac{1}{64} \frac{e^{3}}{n^{2}} \geq 10^{12} e n
$$

if $n$ is large enough. Therefore, we can apply Lemma 3.1 and obtain that

$$
\mathrm{CR}^{*}(G) \geq \frac{\mathrm{CR}^{3}(G)}{(40 e)^{4}} \geq \frac{1}{40^{4}} \cdot \frac{1}{64^{3}} \cdot \frac{e^{9}}{n^{6} e^{4}}=\frac{1}{40^{4} 64^{3}} \frac{e}{n^{2}} \cdot \frac{e^{4}}{n^{4}}>\frac{1}{10^{25}} \frac{e^{4}}{n^{4}}
$$

Case 2: Suppose that $e=e_{0} \geq 4 \cdot 10^{12}(e / n)^{2}$ and $e_{I}<4 \cdot 10^{12}(e / n)^{2}$. Clearly, for any $j<I, e_{j} \geq e_{j+1}$. Let $j<I$ be the greatest index such that $e_{j} \geq 4 \cdot 10^{12}(e / n)^{2}$. Lemma 3.3 implies that $\frac{e_{j}}{n_{j}}>\frac{e}{2 n}>\frac{n^{1 / 3}}{2}$.

We claim that $e_{j} \geq e_{j+1}>e_{j} / 4$. Indeed, by definition, we have

$$
e_{j+1} \geq \frac{e\left(G_{j}^{\prime}\right)}{3}=\frac{e_{j}}{3}\left(1-\frac{10(e / n)^{2 / 3}}{e_{j}^{1 / 3}}-40 \sqrt{\frac{n_{j}}{e_{j}}}\right)>\frac{e_{j}}{4},
$$

provided that $n$ is large enough. Hence, $10^{12}(e / n)^{2} \leq e_{j+1}<4 \cdot 10^{12}(e / n)^{2}$. Thus, we can again apply Lemma 3.3 to obtain $\frac{e_{j+1}}{n_{j+1}}>\frac{e}{2 n}$, so that $n_{j+1}<e_{j+1} \cdot(2 n / e)<$ $4 \cdot 10^{12}(e / n)^{2}(2 n / e)=8 \cdot 10^{12}(e / n)$. The theorem of Ajtai et al. now implies that

$$
\mathrm{CR}\left(G_{j+1}\right) \geq \frac{1}{64} \frac{4^{3} 10^{36}}{8^{2} 10^{24}}\left(\frac{e}{n}\right)^{4}>10^{10} \frac{e^{4}}{n^{4}}
$$

If $n$ is sufficiently large, we can apply Lemma 3.1 to $G_{j+1}$ to conclude that

$$
\mathrm{CR}^{*}(G) \geq \mathrm{CR}^{*}\left(G_{j+1}\right) \geq \frac{10^{30}}{40^{4}} \frac{(e / n)^{12}}{e_{j+1}^{4}} \geq \frac{10^{30}}{40^{4}} \frac{(e / n)^{12}}{400^{4}(e / n)^{8}}>10^{13} \frac{e^{4}}{n^{4}}
$$

Case 3: Suppose now that $e_{I} \geq 4 \cdot 10^{12}(e / n)^{2}$. Since the procedure has stopped, we have $\mathrm{CR}_{I} \geq\left(e_{I} e / n\right)^{4 / 3}+10^{3} e_{I} n_{I}$. We can apply Lemma 3.1 and obtain that

$$
\mathrm{CR}^{*}(G) \geq \mathrm{CR}^{*}\left(G_{I}\right) \geq \frac{1}{40^{4}} \frac{\mathrm{CR}_{I}^{3}}{e_{I}^{4}} \geq \frac{1}{40^{4}} \frac{e^{4}}{n^{4}} .
$$

This concludes the proof of Theorem 1.2. 


\section{References}

1. Ajtai, M., Chvátal, V., Newborn, M., Szemerédi, E.: Crossing-free subgraphs. In: Theory and Practice of Combinatorics. North-Holland Math. Stud., vol. 60, pp. 9-12. North-Holland, Amsterdam (1982)

2. Kleitman, D.: The crossing number of $K_{5, n}$. J. Comb. Theory 9, 315-323 (1970)

3. Leighton, F.T.: Complexity Issues in VLSI: Optimal Layouts for the Shuffle-Exchange Graph and Other Networks. MIT Press, Cambridge (1983)

4. Mohar, B.: Personal communication

5. Mohar, B., Thomassen, C.: Graphs on Surfaces. Johns Hopkins Studies in the Mathematical Sciences. Johns Hopkins University Press, Baltimore (2001)

6. Pach, J., Tóth, G.: Thirteen problems on crossing numbers. Geombinatorics 9, 194-207 (2000)

7. Pach, J., Shahrokhi, F., Szegedy, M.: Applications of the crossing number. Algorithmica 16, 111-117 (1996)

8. Pach, J., Spencer, J., Tóth, G.: New bounds on crossing numbers. Discrete Comput. Geom. 24, 623644 (2000)

9. Székely, L.: Crossing numbers and hard Erdős problems in discrete geometry. Comb. Probab. Comput. 6, 353-358 (1997)

10. Szemerédi, E., Trotter, W.T.: Extremal problems in discrete geometry. Combinatorica 3, 381-392 (1983) 